\title{
Olaparib induces browning of in vitro cultures of human primary white adipocytes
}

\author{
Lilla Nagy ${ }^{\mathrm{a}}$, Boglárka Rauch ${ }^{\mathrm{a}}$, Noémi Balla ${ }^{\mathrm{a}}$, Gyula Ujlaki ${ }^{\mathrm{a}}$, Gréta Kis ${ }^{\mathrm{b}}$, Omar Abdul-Rahman ${ }^{\mathrm{a}}$, \\ Endre Kristóf $^{\mathrm{c}}$, Adrienn Sipos ${ }^{\mathrm{a}}$, Miklós Antal ${ }^{\mathrm{b}}$, Attila Tóth ${ }^{\mathrm{d}, \mathrm{e}}$, Tamás Debreceni ${ }^{\mathrm{f}}$, Ambrus Horváth ${ }^{\mathrm{f}}$, \\ Tamás Maros ${ }^{\mathrm{f}}$, Péter Csizmadia ${ }^{\mathrm{f}}$, Tamás Szerafin ${ }^{\mathrm{f}}$, Péter Bai ${ }^{\mathrm{a}, \mathrm{g}, \mathrm{h}, *}$ \\ ${ }^{a}$ Department of Medical Chemistry, Faculty of Medicine, University of Debrecen, 4032, Hungary \\ ${ }^{\mathrm{b}}$ Department of Anatomy, Histology and Embryology, Faculty of Medicine, University of Debrecen, 4032, Hungary \\ ${ }^{\mathrm{c}}$ Department of Biochemistry and Molecular Biology, Faculty of Medicine, University of Debrecen, 4032, Hungary \\ ${ }^{\mathrm{d}}$ Division of Clinical Physiology, Faculty of Medicine, University of Debrecen, 4032, Hungary \\ ${ }^{\mathrm{e}}$ MTA-DE Research Group of Vascular Biology and Myocardial Pathology, Hungary \\ ${ }_{\mathrm{f}}^{\mathrm{f}}$ Department of Cardiac Surgery, Faculty of Medicine, University of Debrecen, 4032, Hungary \\ ${ }^{\mathrm{g}}$ MTA-DE Lendület Laboratory of Cellular Metabolism, Debrecen, 4032, Hungary \\ ${ }^{\mathrm{h}}$ Research Center for Molecular Medicine, Faculty of Medicine, University of Debrecen, 4032, Hungary
}

\section{A R T I C L E IN F O}

Dataset link: https://figshare.com/s/

78810e13d8ad50b1b58d

Keywords:

Thermogenesis

$\mathrm{NAD}^{+}$

Olaparib

PARP

ARTD

SIRT1

Adipogenesis

Beige adipocytes

White adipocytes

White adipose tissue

Brown adipose tissue

\begin{abstract}
A B S T R A C T
Mitochondrial biogenesis is a key feature of energy expenditure and organismal energy balance. Genetic deletion of PARP1 or PARP2 was shown to induce mitochondrial biogenesis and energy expenditure. In line with that, PARP inhibitors were shown to induce energy expenditure in skeletal muscle. We aimed to investigate whether pharmacological inhibition of PARPs induces brown or beige adipocyte differentiation. SVF fraction of human pericardial adipose tissue was isolated and human adipose-derived mesenchymal stem cells (hADMSCs) were differentiated to white and beige adipocytes. A subset of hADMSCs were differentiated to white adipocytes in the presence of Olaparib, a potent PARP inhibitor currently in clinical use, to induce browning. Olaparib induced morphological changes (smaller lipid droplets) in white adipocytes that is a feature of brown/beige adipocytes. Furthermore, Olaparib induced mitochondrial biogenesis in white adipocytes and enhanced UCP1 expression. We showed that Olaparib treatment inhibited nuclear and cytosolic PAR formation, induced NAD ${ }^{+} / \mathrm{NADH}^{\mathrm{ratio}}$ and consequently boosted SIRT1 and AMPK activity and the downstream transcriptional program leading to increases in OXPHOS. Olaparib treatment did not induce the expression of beige adipocyte markers in white adipocytes, suggesting the formation of brown or brown-like adipocytes.

PARP1, PARP2 and tankyrases are key players in the formation of white adipose tissue. Hereby, we show that PARP inhibition induces the transdifferentiation of white adipocytes to brown-like adipocytes suggesting that PARP activity could be a determinant of the differentiation of these adipocyte lineages.
\end{abstract}

\section{Introduction}

In light of the recent advances the white and brown dichotomy of adipose tissue was revisited upon the discovery of beige adipocytes [1]. Apparently, adipose tissue is a versatile organ that can perform lipid storage, energy expenditure and other biological roles contributing to energy homeostasis to meet the needs of the organism [2,3]. There are two types of adipocytes with considerable oxidative phosphorylation
(OXPHOS) capacity, brown and beige adipocytes that are responsible for facilitating energy expenditure, while white adipocytes are primarily lipid storing cells. Classical brown adipocytes are mitochondriarich multilocular cells in which active OXPHOS and ATP synthesis is uncoupled. These cells are typically found in the interscapular region of newborn humans and around major arteries later in life [4,5]. Unstimulated beige cells reside in white adipose tissue depots and have similar morphology to white adipocytes [6]. Beige adipocytes can

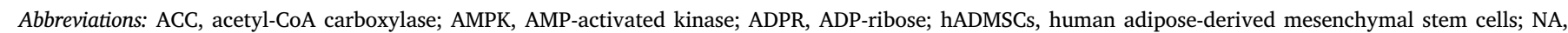

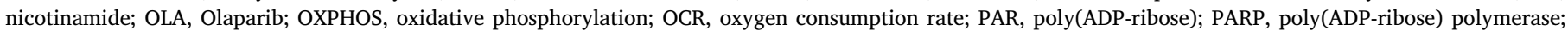
PGC-1 $\alpha$, peroxisome proliferator activated receptor associated cofactor-1 $\alpha$; SVF, stromal vascular fraction; UCP1, uncoupling protein-1

* Corresponding author at: University of Debrecen, Department of Medical Chemistry, 4032 Debrecen, Egyetem tér 1., Hungary.

E-mail address: baip@med.unideb.hu (P. Bai). 
efficiently induce mitochondrial biogenesis in response to adrenergic stimulus in a similar fashion and efficiency as classical brown adipocytes $[1,7]$ that seems protect against obesity and diabetes [8-10].

Poly(ADP-ribose) polymerases (PARPs) represent a 17-member protein family $[11,12]$. Although, PARPs were originally described as DNA repair proteins, recent investigations pointed out that these enzymes also have a significant impact on cellular and organismal metabolism [13] and within that, in regulating mitochondrial metabolism [14-17]. There are several molecular pathways through which PARPs exert their effects on the mitochondria (for review see [14]). From the perspective of the current study, two energy pathways have high relevance. PARPs are enzymes that cleave $\mathrm{NAD}^{+}$to build long branched poly(ADP-ribose) (PAR) chains on target proteins. The vast majority of PARP activity can be attributed to PARP1 and PARP2 [18] and they represent a heavy burden on cellular $\mathrm{NAD}^{+}$levels $[19,20]$. PARP1 and PARP2, therefore, compete with other $\mathrm{NAD}^{+}$-dependent enzymes, such as Sirt1 and, hence, impact on mitochondrial physiology [19-21]. Pharmacological or genetic inhibition of PARP1 or PARP2 induces SIRT1 activity through inducing cellular $\mathrm{NAD}^{+}$levels that leads to mitochondrial biogenesis through activating transcriptional programs driven by peroxisome proliferator activated receptor associated cofactor (PGC)-1 $\alpha$ and FOXO1 [22,23]. PARP1 also has multifaceted interactions with another key energy sensor, AMP-activated kinase (AMPK) [24-27].

PARP-mediated changes to bioenergetics impacted on the (central) nerve system, skeletal, cardiac and smooth muscle, the liver, endothelial cells, cancers and the white and the brown adipose tissue $[13,15,26,28-35]$. Consequently, a plethora of metabolic diseases are associated with the PARP-mediated derailment of bioenergetics $[13,36]$. Genetic deletion of PARP1 can induce heat generation and cold tolerance in mice that is attributed to brown adipocytes [19]. Moreover, the above detailed PARP-mediated elements of the energy homeostasis (e.g. AMPK, Sirt1) were shown to be key mediators of white, brown and beige adipocyte development or function [37-39]. In the current study we set out to assess whether PARP inhibition by Olaparib, a pan-PARP inhibitor [40] can cause a transdifferentiation of white adipocytes to beige or brown adipocytes.

\section{Materials and methods}

\subsection{Chemicals}

Chemicals were obtained from Sigma-Aldrich (St. Louis, MO, USA) unless stated otherwise.

\subsection{Ethical statement}

The study protocol was approved by the Ethics Committee of the University of Debrecen (Hungary) and the National Medical Research Council Committee of Human Reproduction (ETT TUKEB). All experiments were carried out in accordance with the Declaration of Helsinki and the approved ethical guidelines and regulations. Written informed consent from all participants was obtained before the surgical procedure.

\subsection{Isolation, culture and differentiation of hADMSCs}

From human pericardial/mediastinal adipose tissue of patients undergoing a planned cardiac surgery (e.g. valve surgery, coronary bypass surgery, Batista operation) stromal vascular fraction (SVFs) was isolated and from the SVF human adipose-derived mesenchymal stem cells (hADMSCs) were cultivated. No exclusions were applied regarding BMI, age, gender or medications of the patients.

On the day of heart surgery the mediastinal adipose tissue specimens were processed as described in [41]. Samples were digested in PBS with $120 \mathrm{U} / \mathrm{ml}$ collagenase for $1 \mathrm{~h}$ at $37^{\circ} \mathrm{C}$ and filtered through a sieve with a pore size of $100 \mu \mathrm{m}$. Isolated hADMSCs were resuspended in DMEM-F12 HAM medium containing $10 \%$ FBS and seeded to the appropriate vessels. After cell culture reached confluency, differentiation was initiated by using FBS free medium. White adipogenic differentiation was carried out using the protocol described by Fischer-Posovszky and co-workers [42]. To induce beige adipogenic differentiation cells were treated according to Elabd and co-workers [43]. To assess the result of PARP inhibition, white adipocytes were differentiated in the presence of $1 \mu \mathrm{M}$ Olaparib (Selleckchem, Munich, Germany). This concentration was determined by testing our cell model (hADMSCs) with Olaparib for toxicity in a range of $100 \mathrm{nM}-4 \mu \mathrm{M}$ for toxicity. Cells were assayed after 14 days after the induction of differentiation.

\subsection{Microscopic analysis of differentiated hADMSC}

After 2-weeks of differentiation microscopic images were acquired with an OPTIKA XDS-3 trinocular inverted microscope (Science Services, Munich, Germany) using IOS LWD 20X/0.40 $\mathrm{Ph}$ (w.d. $7.66 \mathrm{~mm}$ ) objective at $25^{\circ} \mathrm{C}$ and images were processed using SharpCap 2.8 software.

\subsection{Confocal microscopy}

hADMSCs were seeded on glass coverslips and differentiated as described in 2.3. For the visualization of lipid droplets, on the last day of the differentiation cells were fixed with $4 \%$ formaldehyde for $20 \mathrm{~min}$ at $37^{\circ} \mathrm{C}$ then rinsed three times with $1 \times$ PBS. Following the fixation cells were stained for $20 \mathrm{~min}$ at room temperature with Nile Red dye diluted in $1 x$ PBS in concentration of $10 \mu \mathrm{g} / \mathrm{ml}$. Cells were washed once with PBS and once with water.

On the last day of the differentiation the other set of cells were stained with Mitotracker Red (Thermo Scientific, MA, USA) to assess the mitochondrial network using a working concentration of $100 \mathrm{nM}$ for $30 \mathrm{~min}$ at $37^{\circ} \mathrm{C}$. Cells were washed once with PBS and cells were fixed with $4 \%$ formaldehyde for $20 \mathrm{~min}$ at $37^{\circ} \mathrm{C}$ then rinsed three times with $1 \times$ PBS.

The nuclei were stained with DAPI special formation (NucBlue $^{\circledR}$ Fixed cell Stain ReadyProbes ${ }^{\mathrm{TM}}$ reagent, Life Technologies, Carlesbad, CA, USA) and rinsed in $1 \times$ PBS again.

Confocal images were acquired with a Leica TCS SP8 confocal microscope (Leica Microsystems, Wetzlar, Germany) using an HC PL APO CS2 $63 \times / 1.40$ OIL immersion objective on a DMI6000 CS microscope at $25{ }^{\circ} \mathrm{C}$. The following lasers were applied: OPSL 488 for Mitotracker Red and for Nile Red, and 405 visible for DAPI. Images were processed using LAS X 2.0.1.14392 software. The number of mitochondria was determined using CellProfiler software (The Broad Institute of MIT, MA).

\subsection{Gene expression and RT-qPCR}

Reverse transcription-coupled real time quantitative PCR (RT-qPCR) reactions were performed similarly as in [44]. Briefly, total RNA was prepared using TRIzol reagent (Invitrogen, Carlsbad, CA, USA) according to the manufacturer's instructions, then reverse transcription was performed (High Capacity cDNA Reverse Transcription Kit, Applied Biosystems, Foster City, CA, USA). $10 \times$ diluted cDNA was used for RTqPCR reactions (PCRBIOSYSTEMS, qPCRBIO SyGreen Mix Lo-ROX, London, United Kingdom). The RT-qPCR reactions were performed using the Light-Cycler system (Roche Applied Science, Mannheim, Germany). Primers are summarized in Table 1. Expression was normalized to the geometric mean of three control genes (cyclophyllin, $\beta$ actin, 36B4 or YWAHZ). 
Table 1

Primers used in RT-qPCR reactions.

\begin{tabular}{|c|c|c|c|}
\hline Gene & & Forward primer & Reverse primer \\
\hline 36B4 & Human & 5'-CCATTGAAATCCTGAGTGATGTG-3' & 5'-GTCGAACACCTGCTGGATGAC-3' \\
\hline$\beta$-actin & Human & 5'-GACCCAGATCATGTTTGAGACC-3' & 5'-CATCACGATGCCAGTGGTAC-3' \\
\hline Cyclophilin A & Human & 5'-GTCTCCTTTGAGCTGTTTGCAGAC-3' & 5'-CTTGCCACCAGTGCCATTATG-3' \\
\hline PGC1 $\alpha$ & Human & 5'-TTCCTCTGACCCCAGAGTCACC-3' & 5'-TTGCAAGAGGACTTCAGCTTTGG-3' \\
\hline $\operatorname{PPAR} \gamma$ & Human & 5'-GTGGCCGCAGATTTGAAAGAAG-3' & 5'-CCATGGTCATTTCGTTAAAGGCTG-3' \\
\hline PRDM16 & Human & 5'-CACTGTGCAGGCAGGCTAAGAA-3' & 5'-AGAGGTGGTTGATGGGGTGAAA-3' \\
\hline TBX1 & Human & 5'-TCССАССТTCCAAGTGAAGCTC-3' & 5'-CACGATTTGCTTCATCCACTGC-3' \\
\hline TMEM26 & Human & 5'-ACCTCCCATGTGTGGACATCCT-3' & 5'-ACCAACAGCACCAACAACCTCA-3' \\
\hline SIRT1 & Human & 5'-TGGCAAAGGAGCAGATTAGTAGGC-3' & 5'-TGGACTCTGGCATGTCCCACT-3' \\
\hline UCP1 & Human & 5'-AACGAAGGACCAACGGCTTTC-3' & 5'-GGCACAGTCCATAGTCTGCCTTG-3' \\
\hline YWAHZ & Human & 5'-CCGCCAGGACAAACCAGTAT-3' & 5'-ACTTTTGGTACATTGTGGCTTCAA-3' \\
\hline
\end{tabular}

Table 2

List of antibodies used in the study.

\begin{tabular}{llll}
\hline Primary antibodies & & & \\
\hline B-Actin-Peroxidase (Sigma Aldrich) & mouse & polyclonal & $1: 25000$ \\
ACC (C83B10) (Cell Signaling) & rabbit & monoclonal & $1: 1000$ \\
Phospho-ACC(Serine ${ }^{79}$ ) (Cell Signaling) & rabbit & polyclonal & $1: 1000$ \\
AMPKa (D63G4) (Cell signaling) & rabbit & monoclonal & $1: 1000$ \\
Phospho- AMPKa (Threonine ${ }^{172}$ ) (40H9) (Cell & rabbit & monoclonal & $1: 1000$ \\
$\quad$ signaling) & & & \\
FOXO1 (C29H4) (Cell Signaling) & rabbit & monoclonal & $1: 1000$ \\
Histone H3 (Cell Signaling) & rabbit & polyclonal & $1: 1000$ \\
LaminB1 (Cell signaling) & rabbit & monoclonal & $1: 1000$ \\
PGC1 $\alpha$ (Thermo Fisher Scientific) & rabbit & polyclonal & $1: 1000$ \\
Poly(ADP-ribose) (PAR) (Enzo Life Science) & mouse & monoclonal & $1: 500$ \\
TBX1 (Gene Tex) & rabbit & polyclonal & $1: 500$ \\
UCP1 (Sigma Aldrich) & mouse & monoclonal & $1: 1000$ \\
Secondary antibodies & & & \\
HRP-linked anti-rabbit IgG (Cell Signaling) & goat & polyclonal & $1: 2000$ \\
Peroxidase conjugated anti-mouse IgG (Sigma & rabbit & polyclonal & $1: 80000$ \\
$\quad$ Aldrich) & & & \\
\hline
\end{tabular}

\subsection{Protein extraction and Western blotting}

The isolation of cytoplasmic and nuclear fraction of proteins from hADMSCs and the subsequent Western blotting are described in [45]. Blots were probed with the antibodies summarized in Table 2.

Signals were detected using enhanced chemiluminescence (ECL) and were captured by ChemiDoc Touch (Bio-Rad Laboratories, CA, USA).

\subsection{Determination of $N A D^{+} / N A D H$ ratio}

NAD/NADH Quantification Kit (Sigma) was used for the determination of $\mathrm{NAD}^{+} / \mathrm{NADH}$ ratio following the manufacturer's instructions. $\mathrm{NAD}^{+}$and $\mathrm{NADH}$ content was normalized for protein content.

\subsection{Determination of the size and number of lipid droplets}

Preadipocytes were seeded and differentiated on PerkinElmer 96well black clear bottom plate. On the last day of the differentiation cells were fixed with $4 \%$ formaldehyde for $20 \mathrm{~min}$ at $37^{\circ} \mathrm{C}$ then rinsed two times with $1 \times$ PBS. Following the fixation cells were stained with Nile Red dye using a working concentration of $10 \mu \mathrm{g} / \mathrm{ml}$ and diluted in $1 \times$ PBS for $20 \mathrm{~min}$ at room temperature (diluted from $10 \mathrm{mg} / \mathrm{ml}$ stock solution). The dye charges the lipid droplets enabling the visualization of the pattern and size of lipid droplets in adipocytes. Cells were washed once with PBS and once with water. The nuclei were stained with DAPI special formation (NucBlue $^{\circledR}$ Fixed cell Stain ReadyProbes ${ }^{\mathrm{TM}}$ reagent, Life Technologies, Carlesbad, CA, USA) and rinsed in $1 \times$ PBS again. Images were acquired with an Opera Phenix ${ }^{\mathrm{TM}}$ High-Content Screening System (PerkinElmer, Waltham, MA, USA) $40 \times$ water immersion objective at $25^{\circ} \mathrm{C} .488 \mathrm{~nm}$ laser was used for the detection of Nile Red and $405 \mathrm{~nm}$ laser for DAPI. Images were processed using Harmony software and analyzed by Image $J$ software [46]. We calculated the ratio of differentiation by counting the cells containing at least two large lipid droplets (differentiated) and those that did not (undifferentiated).

\subsection{Determination of oxygen consumption (OCR)}

Oxygen consumption rate was determined using XF96 Flux Analyzer (Agilent Technologies, CA, USA). hADMSCs were seeded in 96-well assay plates, then differentiated as described previously. After recording the baseline oxygen consumption, cells received a single bolus dose of dibutyril-cAMP $(500 \mu \mathrm{M}$ final concentration) simulating adrenergic stimulation. Then OCR was recorded every $5 \mathrm{~min}$ for $30 \mathrm{~min}$. As a last step, cells received a single bolus dose of antimycin A $(10 \mu \mathrm{M})$ and rotenone $(2.5 \mu \mathrm{M})$ for distinguishing the mitochondrial from non-mitochondrial oxygen consumption. After the measurement cells were fixed and Sulphorhodamine B assay was used for the determination of total protein content. OCR values were normalized to protein content and normalized readings were analyzed and plotted.

\subsection{Immunoprecipitation}

Cells were lysed in RIPA lysis buffer (50 mM Tris, $150 \mathrm{mM} \mathrm{NaCl}$, $0.1 \%$ SDS, $1 \%$ Triton X 100, $0.5 \%$ sodium deoxycolate, 1 mM EDTA, $1 \mathrm{mM} \mathrm{Na} \mathrm{VO}_{4}, 1 \mathrm{mM} \mathrm{NaF}$ and protease inhibitor cocktail). FOXO1 acetylation levels were analyzed by immunoprecipitation from lysates with anti-FOXO1 (1:1000, Cell Signalling, Danvers, MA, USA) followed by Western blot using an acetyl-lysine antibody (1:500, Cell Signalling) that was normalized to total FOXO1 levels.

\subsection{Statistical analysis}

Throughout the text $\mathrm{n}$ refers to the number of donors (biological replicates) assessed. All data is represented as average \pm SEM, unless stated otherwise. Data were analyzed by using GraphPad Prism 3 software and statistical analysis was performed by using one-way ANOVA followed by Dunnett's post-hoc test.

\section{Results}

\subsection{Olaparib induces mitochondrial biogenesis in white adipocytes}

In the study we used white adipose tissue-derived stem cells (hADMSCs) that were differentiated into white or beige adipocytes; a subset of the progenitor cells that differentiated into white adipocytes received OLA, a potent PARP inhibitor that is used in the clinical practice. First, we assessed the morphology of the cells, as in previous studies smaller lipid droplets were characteristic for beige differentiation [47-49]. Beige adipocytes and OLA-treated white adipocytes had 

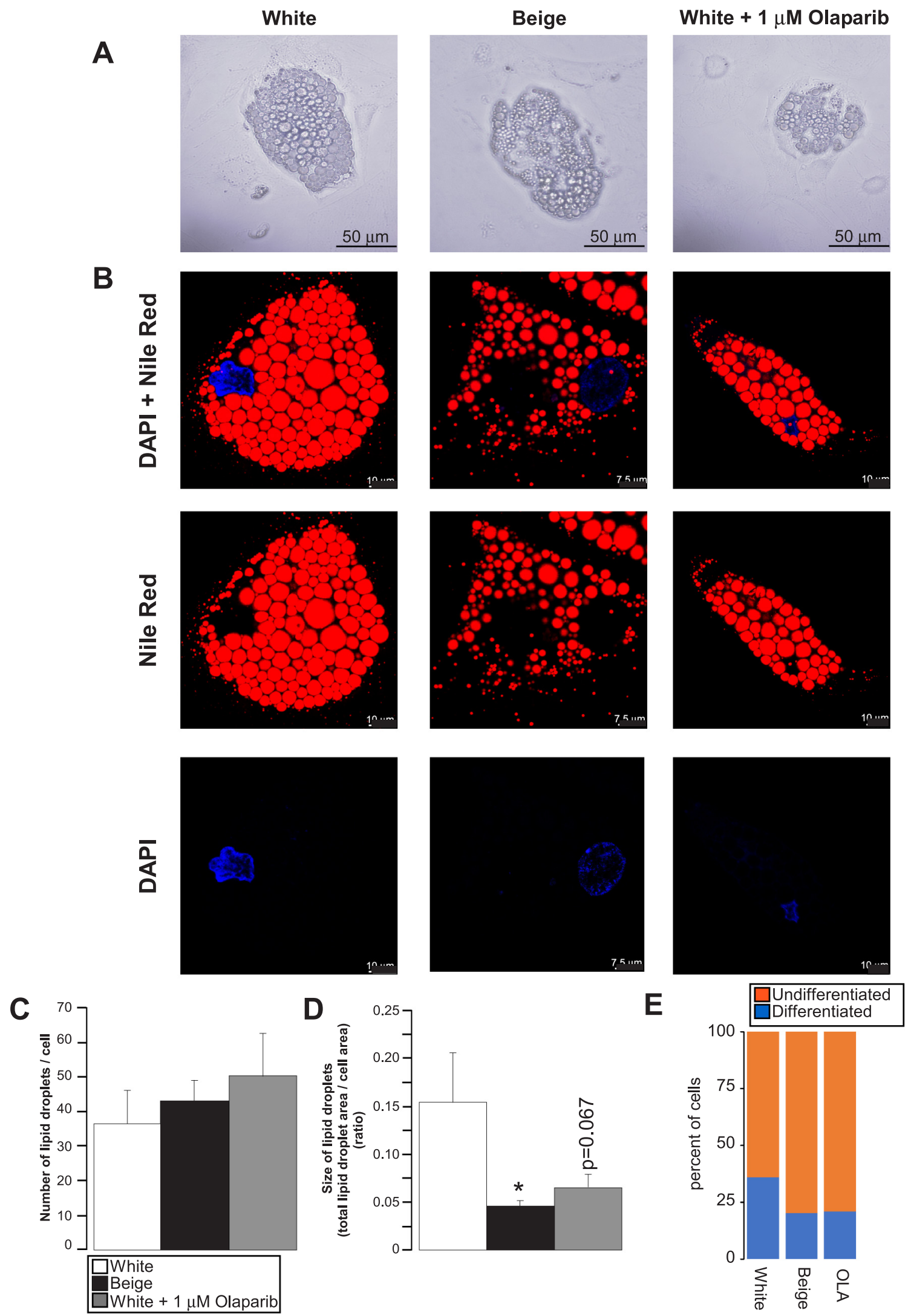

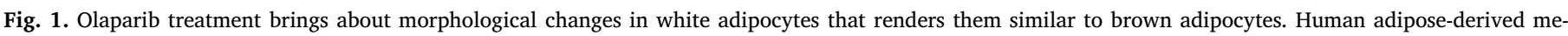

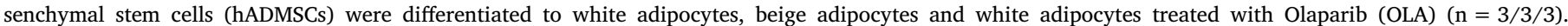

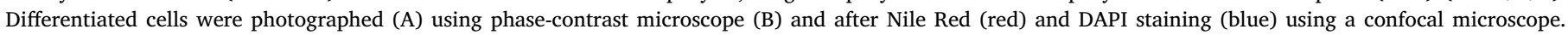

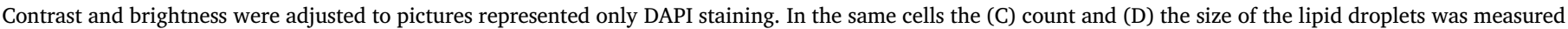

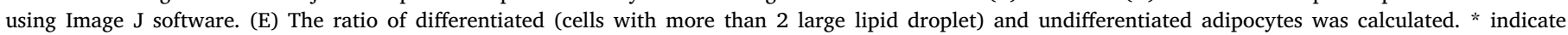

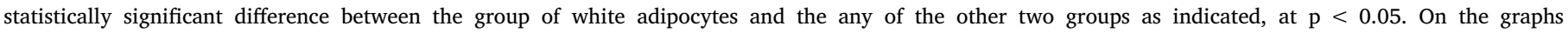
average \pm SEM was plotted. 
A
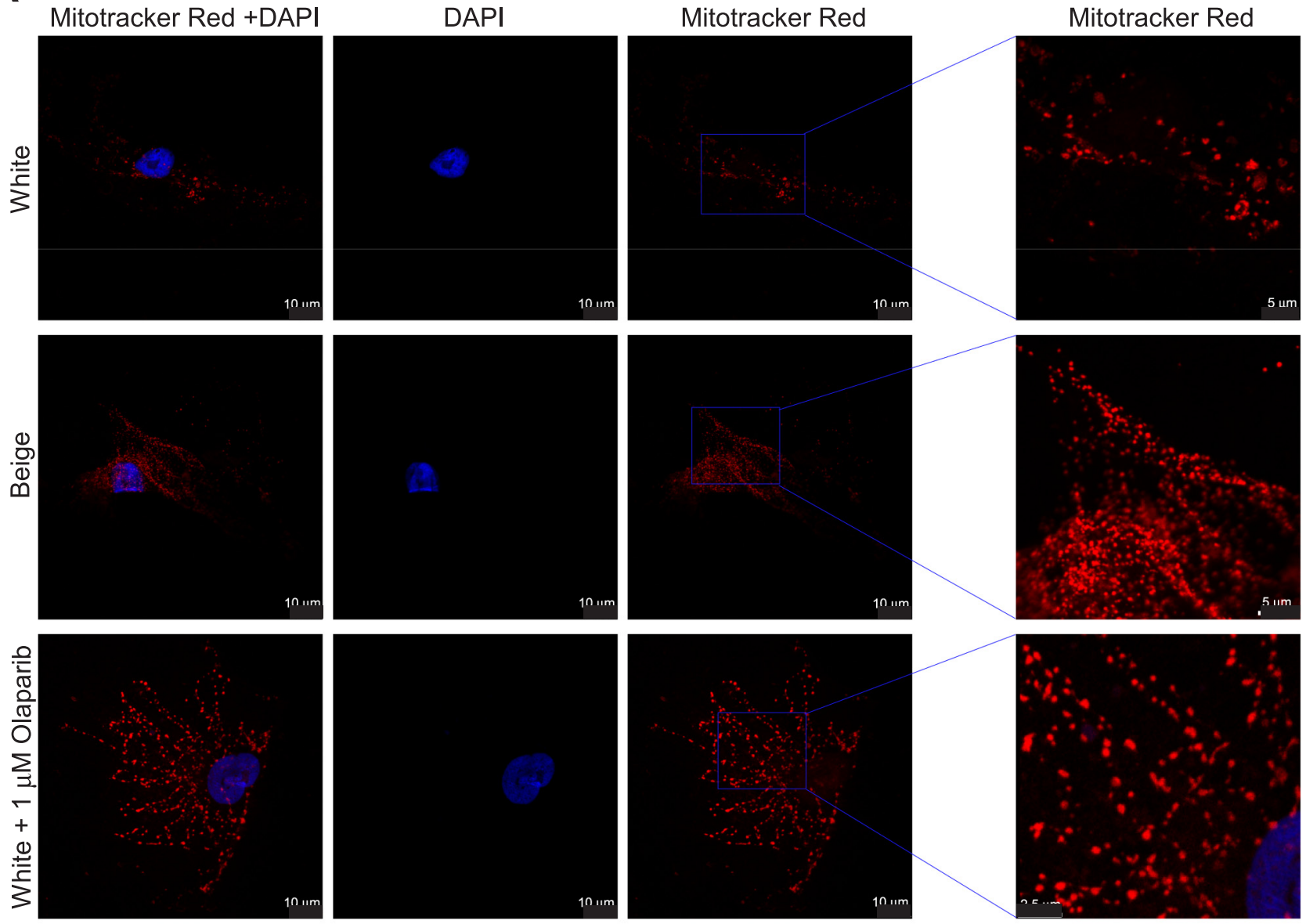

B

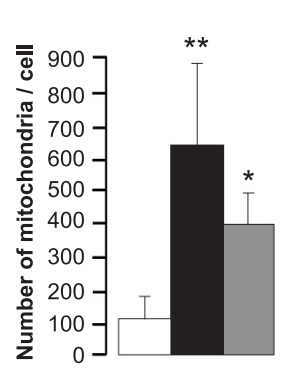

C
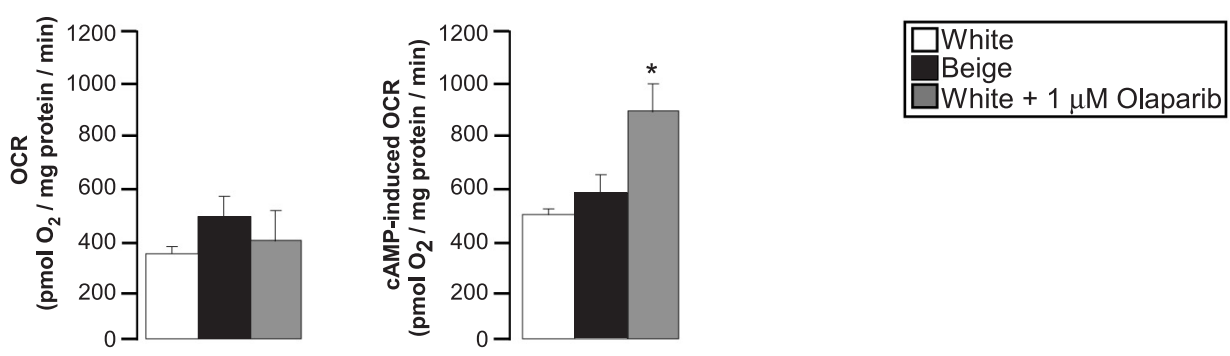

D
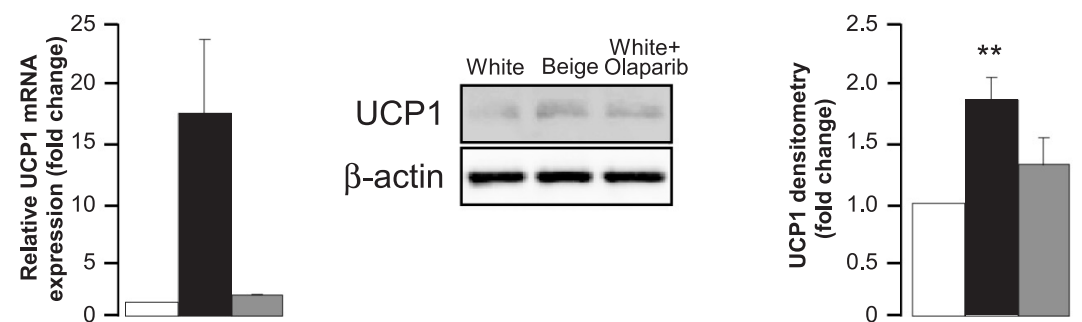

Fig. 2. Olaparib treatment induces browning in white adipocytes. Human adipose-derived mesenchymal stem cells (hADMSCs) were differentiated to white adipocytes, beige adipocytes and white adipocytes treated with Olaparib (OLA). (A) Differentiated cells were charged with Mitotracker Red (in red) and the nuclei were visualized by DAPI (in blue). (B) These confocal pictures were further analyzed by using CellProfiler and the number of mitochondria was determined. (C) Basal and cAMP-induced cellular oxygen consumption rate (OCR) were determined by Seahorse extracellular flux analyzer $(n=3)$. (D) UCP1 mRNA levels ( $n=5)$ by RT-qPCR and protein levels in cytoplasmic fraction by western blotting $(n=5)$ were determined. A representative blot is shown. * and ** indicate statistically significant difference between the group of white adipocytes and the any of the other two groups as indicated, at $\mathrm{p}<0.05$ or $\mathrm{p}<0.01$, respectively. On the graphs average \pm SEM was plotted. 
smaller lipid droplets as compared to white adipocytes (Fig. 1A-D). We assessed the rate of differentiation, too. Beige differentiation and OLA treatment decreased the rate of differentiation as compared to white adipocytes (Fig. 1E).

Next, we assessed mitochondrial content using Mitotracker red staining. Beige adipocytes had markedly higher mitochondrial content than white adipocytes (Fig. 2A, B). OLA treatment also significantly induced mitochondrial content in white adipocytes (Fig. 2A, B). Increases in mitochondrial content was translated into increased mitochondrial oxygen consumption rates (OCR) in beige adipocytes and in OLA-treated white adipocytes when induced by a membrane-permeable cAMP analogue ( $500 \mu \mathrm{M}$ dibutyril-cAMP), mimicking adrenergic stimulus (Fig. 2C). Finally, we assessed the expression of uncoupling protein-1 (UCP1), the major molecular marker of brown and beige adipocytes which is responsible for mitochondrial uncoupling. UCP1 mRNA and protein expression was induced in beige adipocytes and OLA-treated white adipocytes as compared to white adipocytes (Fig. 2D).

Taken together, OLA, similarly to the previous observations [19-21,50,51], induces mitochondrial biogenesis and probably uncoupling in our cellular model.

\subsection{Olaparib treatment induces Sirt1 and AMPK}

We assessed the molecular pathways that can be responsible for the induction of mitochondrial biogenesis. Sirt1 activation had been linked to PARP inhibition by numerous prior studies [19,21,50-53] and Sirt1 activation was shown to play a major role in beige and brown differentiation [54-56]. OLA inhibited PARP activity in the nuclear and cytosolic fractions (Fig. 3A). Interestingly, beige differentiation decreased cytosolic PAR formation (Fig. 3A). Decreased PARP activity slightly increased $\mathrm{NAD}^{+}$levels, significantly reduced NADH levels that culminated in an increased $\mathrm{NAD}^{+} / \mathrm{NADH}$ ratio in beige adipocytes and OLAtreated white adipocytes (Fig. 3B) that is a known activator of SIRT1 [57]. We next assessed the expression of Sirt1 and its downstream targets. Interestingly, in beige adipocytes we observed decreased the expression of SIRT1 (Fig. 3C) and FOXO1 (Fig. 3D), while PGC1 $\alpha$ protein expression was drastically induced (Fig. 3F) when compared to white adipocytes. OLA treatment did not change the expression of SIRT1, FOXO1, in turn, PGC1 $\alpha$ expression was induced as compared to the white adipocyte control (Fig. 3C-D, F). Importantly, OLA treatment decreased the acetylation of FOXO1 suggesting increased SIRT1 activity (Fig. 3F).

Another pathway that can induce mitochondrial biogenesis is AMPK. We assessed AMPK activity through assessing key phosphorylation events. AMPK is a trimeric enzyme that is phosphorylated upon activation at Threonine ${ }^{172}$ of the $\alpha$ subunit [58], AMPK phosphorylation levels at $\alpha$ Threonine ${ }^{172}$ were higher in beige cells as compared to white adipocytes (Fig. 4A). Acetyl-CoA carboxylase (ACC) is phosphorylated by AMPK at Serine ${ }^{79}$ and is, therefore, considered a suitable marker of AMPK activation. ACC phosphorylation were induced drastically in beige as compared to white adipocytes (Fig. 4B). AMPK phosphorylation was induced upon OLA treatment in white adipocytes to similar levels as in beige adipocytes (Fig. 4A) and we observed trend for higher pACC levels, too (Fig. 4B).

\subsection{PARP inhibition yields brown-like adipocytes}

Finally, we assessed markers of beige adipocyte differentiation, PRDM16, TMEM26, PPAR $\gamma 1$ and TBX1 $[1,59,60]$. The expression of all markers were higher in beige adipocytes than in white adipocytes (Fig. 5). Nevertheless, the expression of these markers was not induced in OLA-treated white adipocytes as compared to control white adipocytes (Fig. 5).

\section{Discussion}

In this study we provided evidence that Olaparib (OLA), induces mitochondrial biogenesis and, hence, browning in human white adipocytes. OLA is a pan-PARP inhibitor, inhibiting all members of the PARP family [40]. PARP1 [15,19,20,27,28,50,51,61-63], PARP2 [20,21,31], PARP7 [64], PARP10 [34], tankyrases [65] are all implicated in regulating mitochondrial metabolism. The vast majority of PARP activity is attributed to PARP1 and PARP2 [18,31,66] making it likely that PARP1 and PARP2 are responsible for mitochondrial biogenesis upon OLA treatment. Nevertheless, the drastic inhibition of PARP activity in the cytoplasm suggest the involvement of other, yet uncovered, PAR(P)-dependent pathways in OLA-elicited effects. It is of note that studies using other, structurally different PARP inhibitors are necessary to validate our observations in the context of beige/brown differentiation, however, in different model systems other pharmacological PARP inhibitors and genetic PARP inhibition was shown to induce mitochondrial biogenesis [15,19,20,27,28,50,51,61-63].

The involvement of PARPs in mitochondrial physiology was first shown in 1998 by Virág et al. [17], since then a plethora of pathways converging on mitochondrial metabolism were shown to be PARP-dependent (reviewed in [14]). In this study we found the upregulation of two pathways by OLA, namely, the induction of SIRT1 and AMPK.

Pharmacological PARP inhibition was shown to increase $\mathrm{NAD}^{+}$levels and hence induce SIRT1 that subsequently drives mitochondrial biogenesis in skeletal muscle $[19,50]$. Our study extends these observations by showing that brown adipose tissue is also a subject of induction of mitochondrial biogenesis by PARP inhibition. While previous studies showed the upregulation of SIRT1 activity in beige differentiation [37-39,56,67], in our model (SVF from pericardial adipose tissue), in beige adipocytes we found a downregulation of the expression of SIRT1 and FOXO1, nevertheless, SIRT1 activity was induced. These changes are puzzling in light of the previous literature, nevertheless, can be an intrinsic feature of the pericardial adipose tissue, indeed, the metabolic behavior of the adipose tissue from different anatomical locations is known [68-70] and mediastinal/pericardial adipose tissue stem cells exhibit beige characteristics [71] that may explain discrepancies between other studies [38,39,48,72,73]. Nonetheless, OLA treatment readily induced SIRT1 activity and its downstream targets enabling efficient induction of mitochondrial biogenesis.

AMPK activation was very pronounced in beige and OLA-treated white adipocytes as compared to untreated white adipocytes. AMPK was shown to be key player in driving brown adipose tissue and beige adipocyte differentiation and function [38,39,72-74]. AMPK is an upstream regulator of SIRT1 [75] suggesting close collaboration between the two pathways. AMPK and PARP1 has an intricate and multifaceted interactions [14,24-27].

When we assessed the differentiation markers, PRDM16, PPAR $\gamma 1$ and TBX1 did not show major increase in OLA-treated white adipocytes as compared to beige adipocytes. Nevertheless, OLA-treatment induced browning, characterized by mitochondrial biogenesis and increases in UCP1 expression, features of brown and beige adipocytes. These data make it likely that OLA rather induces the differentiation of brown or brown-like adipocytes than true beige cells. The other explanation could be, as mentioned earlier, that mediastinal/pericardial adipose tissue stem cells inherently exhibit beige characteristics [71], therefore, beige characteristics cannot be further induced in these cells.

In this study we show that inhibition PARP enzyme activity induces browning in white adipocytes. A large set of previous studies have shown that PARP1 and PARP2 are necessary for efficient white adipocyte differentiation $[35,65,76-80]$. In good agreement with that, OLA treatment and beige differentiation decreased the rate of differentiation. It is tempting to speculate that PARP activation could be vital for making the decision on the course of adipocyte differentiation between the white and brown/beige lineages. Along the same logic, as high fat feeding can induce PARP activity [19], in obesity or in insulin 

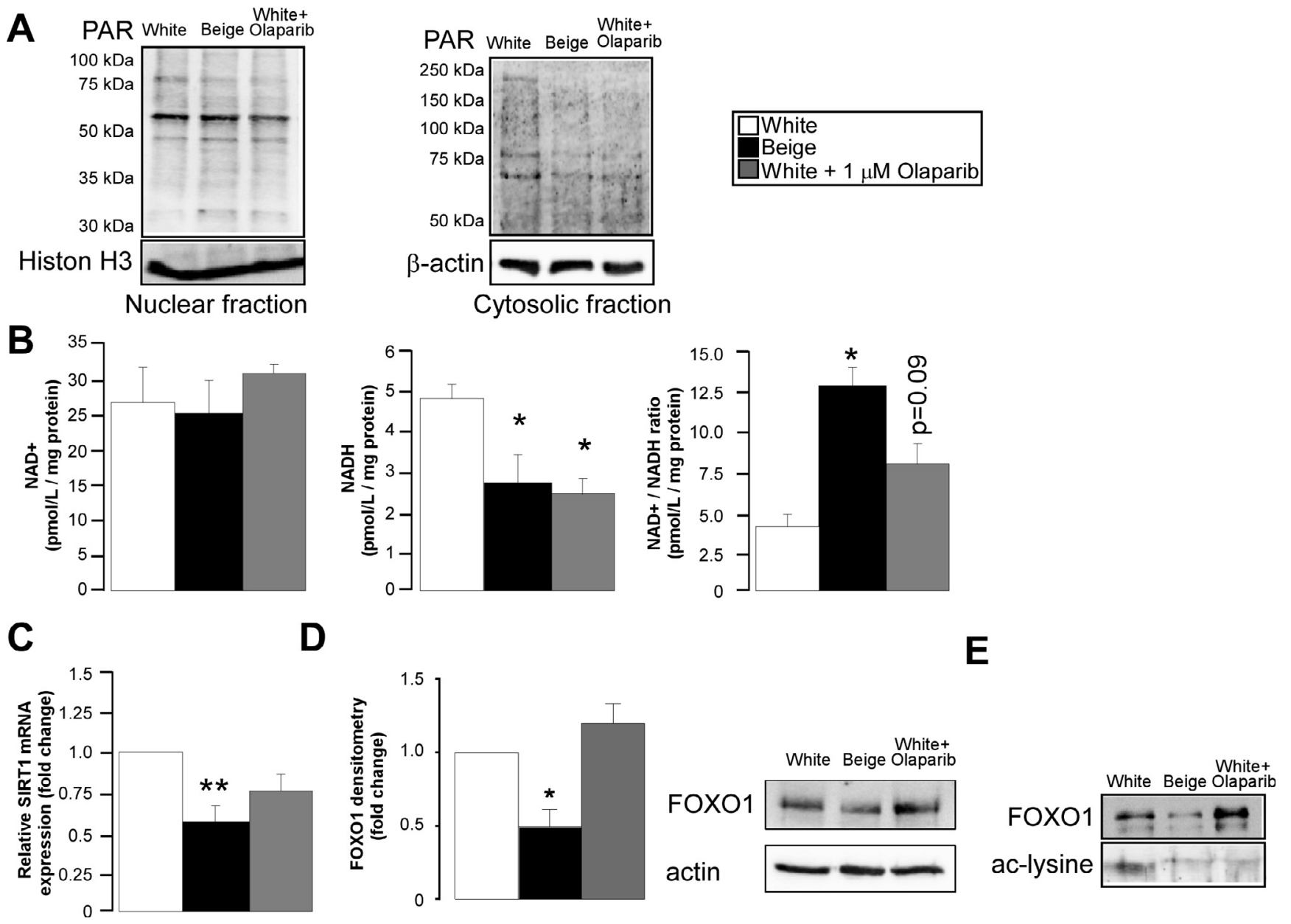

Fig. 3. PARP inhibition induces SIRT1 in white adipocytes. Human adipose-derived mesenchymal stem cells (hADMSCs) were differentiated to white adipocytes, beige adipocytes and white adipocytes treated with Olaparib (OLA). (A) PAR levels were assessed in the nucleus and in the cytosol by Western blotting. Representative images are shown. (B) Cellular $\mathrm{NAD}^{+}$and NADH content, as well as NAD ${ }^{+} / \mathrm{NADH}$ ratio were determined by using NAD/NADH Quantification Kit $(n=5)$. (C) SIRT1 mRNA levels were determined by RT-qPCR $(n=5)$. (D) FOXO1 protein levels were determined by Western blotting ( $n=5)$. A representative blot is shown. A representative blot is shown. (E) FOXO1 acetylation levels were determined by immunoprecipitating FOXO1 and probing the immunoprecipitated protein for FOXO1 and acetyl-lysine in Western blot $(n=1) . *$ and ** indicate statistically significant difference between the group of white adipocytes and the any of the other two groups as indicated, at $\mathrm{p}<0.05$ or $\mathrm{p}<0.01$, respectively. On the graphs average \pm SEM was plotted.

A
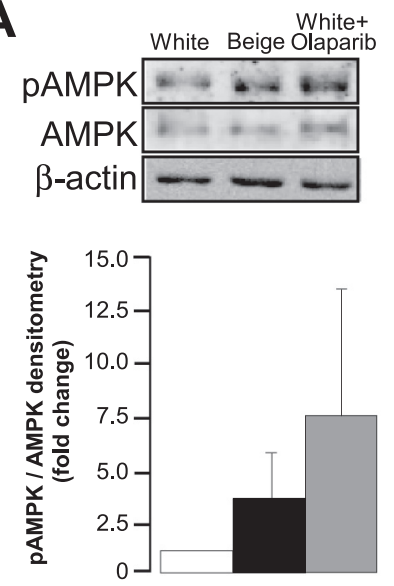

B
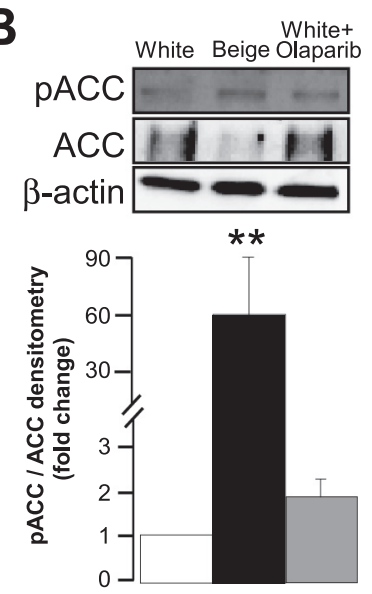

Fig. 4. Olaparib induces AMPK in white adipocytes. Human adipose-derived mesenchymal stem cells (hADMSCs) were differentiated to white adipocytes, beige adipocytes and white adipocytes treated with Olaparib (OLA), then (A) AMPK phosphorylation on Threonine ${ }^{172}(n=4)(B)$ and ACC phosphorylation on Serine ${ }^{79}$ were investigated in western blot measurements $(n=3)$. On the graphs average \pm SEM was plotted. A representative images are shown. resistance PARP activation may hamper brown or beige adipocytes deregulating organismal energy balance and, hence, may play pathophysiological role in these diseases.

\section{Data availability}

All primary data is uploaded to https://figshare.com/s/ 78810e13d8ad50b1b58d (DOI: 10.6084/m9.figshare.7868417)

\section{Acknowledgments}

We are grateful for Mr. László Finta and Mr. László Bancsi for the technical assistance (Dept. Medical Chemistry, UD). Our work was supported by grants from NKFIH (K123975, FK128387, PD124110, PD116262), by GINOP-2.3.2-15-2016-00006 and GINOP-2.3.2-152016-00050, financed by the Hungarian Government and co-financed by the European Structural Fund, EFOP-3.6.2-16-2017-0006 financed by the Hungarian Government and co-financed by the European Social Fund, the Momentum fellowship and the PROJEKT2017-44 grant of the Hungarian Academy of Sciences and the University of Debrecen and Campus France. EK was supported by the János Bolyai Fellowship of the Hungarian Academy of Sciences and the ÚNKP-18-4 New National Excellence Program of the Ministry of Human Capacities in Hungary. 
A

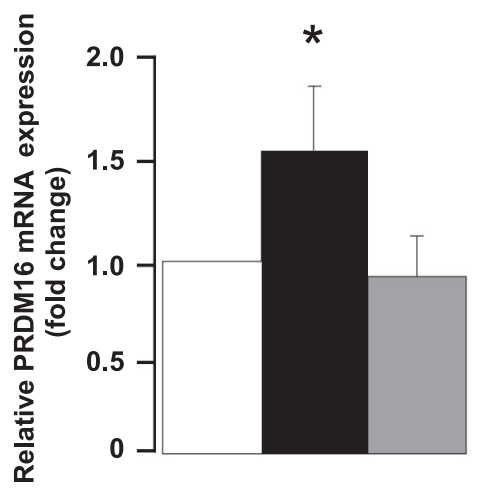

D

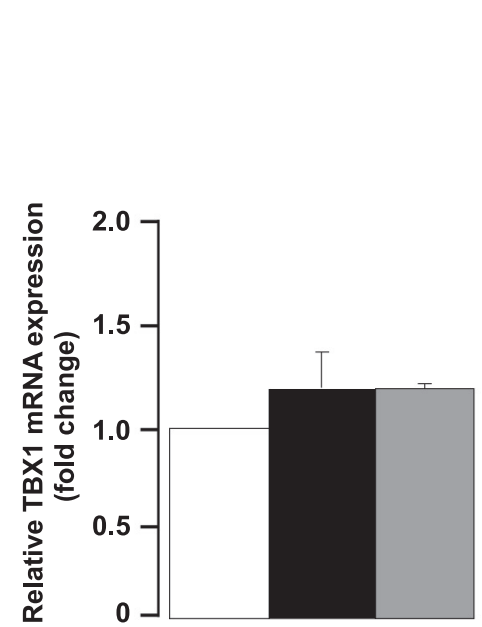

B
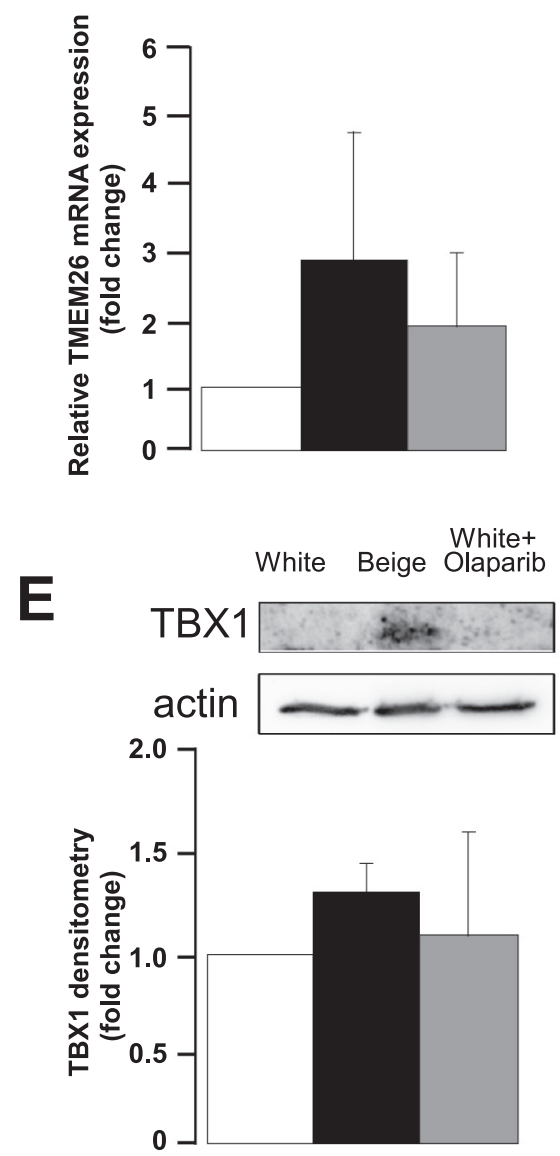
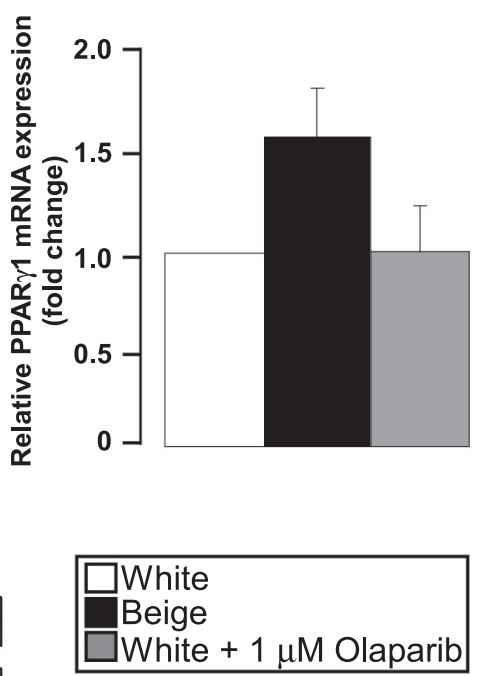

Fig. 5. Markers of beige adipocyte differentiation. Human adipose-derived mesenchymal stem cells (hADMSCs) were differentiated to white adipocytes, beige adipocytes and white adipocytes treated with Olaparib (OLA), then the mRNA levels of (A) PRDM16 $(n=3)$, (B) TMEM26 $(n=4)$, (C) PPAR $\gamma 1$ ( $n=1$, and the error bars represent \pm SD) and (D) TBX1 ( $n=3$ ) were assayed in RT-qPCR measurements. (E) In the same cells TBX1 protein levels were assessed by Western blotting in nuclear fraction $(\mathrm{n}=3)$. A representative blot is shown. *,**and $* * *$ indicate statistically significant difference between the group of white adipocytes and the other two groups, beige and Olaparib-treated white adipocytes, at $\mathrm{p}<0.05, \mathrm{p}<0.01$ or $\mathrm{p}<0.001$, respectively. On the graphs average \pm SEM was plotted except for $\operatorname{PPAR} \gamma 1$, as indicated above.

\section{Authorship contributions}

Performed experiments: LN, BR, NB, GU, GK, OAR.

Analyzed data: LN, BR, NB, GU, GK, OAR.

Provided human samples: TS, TD, AH, TM, PC.

Wrote the manuscript: EK, MA, AT, PB.

\section{Conflict of interest statement}

The authors have no competing financial or non-financial interests to declare.

\section{References}

[1] J. Wu, P. Bostrom, L.M. Sparks, L. Ye, J.H. Choi, A.H. Giang, et al., Beige adipocytes are a distinct type of thermogenic fat cell in mouse and human, Cell 150 (2) (2012) $366-376$.

[2] A. Giordano, A. Smorlesi, A. Frontini, G. Barbatelli, S. Cinti, White, brown and pink adipocytes: the extraordinary plasticity of the adipose organ, Eur. J. Endocrinol. 170 (5) (2014) R159-R171.

[3] P. Cohen, B.M. Spiegelman, Cell biology of fat storage, Mol. Biol. Cell 27 (16) (2016) 2523-2527.

[4] J. Nedergaard, B. Cannon, Brown adipose tissue as a heat-producing thermoeffector, Handb. Clin. Neurol. (2018) 156137-156142.

[5] M.E. Lidell, M.J. Betz, O. Dahlqvist Leinhard, M. Heglind, L. Elander, M. Slawik, et al., Evidence for two types of brown adipose tissue in humans, Nat. Med. 19 (5) (2013) 631-634.

[6] S. Altshuler-Keylin, K. Shinoda, Y. Hasegawa, K. Ikeda, H. Hong, Q. Kang, et al.,
Beige adipocyte maintenance is regulated by autophagy-induced mitochondrial clearance, Cell Metab. 24 (3) (2016) 402-419.

[7] M. Harms, P. Seale, Brown and beige fat: development, function and therapeutic potential, Nat. Med. 19 (10) (2013) 1252-1263.

[8] M. Claussnitzer, S.N. Dankel, K.H. Kim, G. Quon, W. Meuleman, C. Haugen, et al., FTO obesity variant circuitry and adipocyte browning in humans, N. Engl. J. Med. 373 (10) (2015) 895-907.

[9] M. Alcala, M. Calderon-Dominguez, D. Serra, L. Herrero, M. Viana, Mechanisms of impaired brown adipose tissue recruitment in obesity, Front. Physiol. 10 (2019), https://doi.org/10.3389/fphys.2019.00094.

[10] P.E. Scherer, The many secret lives of adipocytes: implications for diabetes, Diabetologia 62 (2) (2019) 223-232.

[11] M.O. Hottiger, P.O. Hassa, B. Luscher, H. Schuler, F. Koch-Nolte, Toward a unified nomenclature for mammalian ADP-ribosyltransferases, Trends Biochem. Sci. 35 (4) (2010) 208-219.

[12] J.C. Ame, C. Spenlehauer, G. de Murcia, The PARP superfamily, BioEssays 26 (8) (2004) 882-893.

[13] A. Vida, J. Marton, E. Miko, P. Bai, Metabolic roles of poly(ADP-ribose) polymerases, Semin. Cell Dev. Biol. (2017) 63135-63143.

[14] P. Bai, L. Nagy, T. Fodor, L. Liaudet, P. Pacher, Poly(ADP-ribose) polymerases as modulators of mitochondrial activity, Trends Endocrinol. Metab. 26 (2) (2015) 75-83.

[15] S.A. Andrabi, G.K. Umanah, C. Chang, D.A. Stevens, S.S. Karuppagounder, J.P. Gagne, et al., Poly(ADP-ribose) polymerase-dependent energy depletion occurs through inhibition of glycolysis, Proc. Natl. Acad. Sci. U.S.A. 111 (28) (2014) 10209-10214.

[16] K. Modis, D. Gero, K. Erdelyi, P. Szoleczky, D. Dewitt, C. Szabo, Cellular bioenergetics is regulated by PARP1 under resting conditions and during oxidative stress, Biochem. Pharmacol. 83 (5) (2012) 633-643.

[17] L. Virag, A.L. Salzman, C. Szabo, Poly(ADP-ribose) synthetase activation mediates mitochondrial injury during oxidant-induced cell death, J. Immunol. 161 (7) (1998) 3753-3759.

[18] G. Zarkovic, E.A. Belousova, I. Talhaoui, C. Saint-Pierre, M.M. Kutuzov, 
B.T. Matkarimov, et al., Characterization of DNA ADP-ribosyltransferase activities of PARP2 and PARP3: new insights into DNA ADP-ribosylation, Nucl. Acids Res. 46 (5) (2018) 2417-2431.

[19] P. Bai, C. Canto, H. Oudart, A. Brunyanszki, Y. Cen, C. Thomas, et al., PARP-1 inhibition increases mitochondrial metabolism through SIRT1 activation, Cell Metab. 13 (4) (2011) 461-468.

[20] J.S. Mohamed, A. Hajira, P.S. Pardo, A.M. Boriek, MicroRNA-149 inhibits PARP-2 and promotes mitochondrial biogenesis via SIRT-1/PGC-1alpha network in skeletal muscle, Diabetes 63 (5) (2014) 1546-1559.

[21] P. Bai, C. Canto, A. Brunyanszki, A. Huber, M. Szanto, Y. Cen, et al., PARP-2 reg ulates SIRT1 expression and whole-body energy expenditure, Cell Metab. 13 (4) (2011) 450-460.

[22] M.C. Haigis, D.A. Sinclair, Mammalian sirtuins: biological insights and disease relevance, Annu. Rev. Pathol. (2010) 5253-5295.

[23] M.N. Sack, T. Finkel, Mitochondrial metabolism, sirtuins, and aging, Cold Spring Harb. Perspect. Biol. (2012), https://doi.org/10.1101/cshperspect.a013102.

[24] J. Zhou, S. Ng, Q. Huang, Y.T. Wu, Z. Li, S.Q. Yao, et al., AMPK mediates a prosurvival autophagy downstream of PARP-1 activation in response to DNA alkylating agents, FEBS Lett. 587 (2) (2012) 170-177.

[25] B. Gongol, T. Marin, I.C. Peng, B. Woo, M. Martin, S. King, et al., AMPKalpha2 exerts its anti-inflammatory effects through PARP-1 and Bcl-6, Proc. Natl. Acad. Sci. U.S.A. 110 (8) (2013) 3161-3166.

[26] F. Salomone, I. Barbagallo, J. Godos, V. Lembo, W. Currenti, D. Cina, et al., Silibinin restores $\mathrm{NAD}(+)$ levels and induces the SIRT1/AMPK pathway in non-alcoholic fatty liver, Nutrients 9 (10) (2017), https://doi.org/10.3390/nu9101086.

[27] N.B. Fakouri, J.A. Durhuus, C.E. Regnell, M. Angleys, C. Desler, M.M. Hasan-Olive, et al., Rev1 contributes to proper mitochondrial function via the PARP-NAD (+)-SIRT1-PGC1alpha axis, Sci. Rep. 7 (1) (2017) 12480.

[28] S.W. Yu, H. Wang, M.F. Poitras, C. Coombs, W.J. Bowers, H.J. Federoff, et al., Mediation of poly(ADP-ribose) polymerase-1-dependent cell death by apoptosisinducing factor, Science 297 (5579) (2002) 259-263.

[29] M. Chen, Z. Zsengeller, C.Y. Xiao, C. Szabo, Mitochondrial-to-nuclear translocation of apoptosis-inducing factor in cardiac myocytes during oxidant stress: potential role of poly(ADP-ribose) polymerase-1, Cardiovasc. Res. 63 (4) (2004) 682-688.

[30] C.Y. Xiao, M. Chen, Z. Zsengeller, C. Szabo, Poly(ADP-ribose) polymerase contributes to the development of myocardial infarction in diabetic rats and regulates the nuclear translocation of apoptosis-inducing factor, J. Pharmacol. Exp. Ther. 310 (2) (2004) 498-504.

[31] M. Szanto, I. Rutkai, C. Hegedus, A. Czikora, M. Rozsahegyi, B. Kiss, et al., Poly (ADP-ribose) polymerase-2 depletion reduces doxorubicin-induced damage through SIRT1 induction, Cardiovasc. Res. 92 (3) (2011) 430-438.

[32] X. Du, T. Matsumura, D. Edelstein, L. Rossetti, Z. Zsengeller, C. Szabo, et al., Inhibition of GAPDH activity by poly(ADP-ribose) polymerase activates three major pathways of hyperglycemic damage in endothelial cells, J. Clin. Invest. 112 (7) (2003) 1049-1057.

[33] J. Michels, F. Obrist, M. Castedo, I. Vitale, G. Kroemer, PARP and other prospective targets for poisoning cancer cell metabolism, Biochem. Pharmacol. 5 (14) (2014) 00516-524.

[34] J. Marton, T. Fodor, L. Nagy, A. Vida, G. Kis, A. Brunyanszki, et al., PARP10 (ARTD10) modulates mitochondrial function, PLoS One 13 (1) (2018) e0187789.

[35] K.W. Ryu, T. Nandu, J. Kim, S. Challa, R.J. DeBerardinis, W.L. Kraus, Metabolic regulation of transcription through compartmentalized $\mathrm{NAD}(+)$ biosynthesis, Science (2018), https://doi.org/10.1126/science.aan5780.

[36] P. Bai, C. Canto, The role of PARP-1 and PARP-2 enzymes in metabolic regulation and disease, Cell Metab. 16 (5) (2012) 290-295.

[37] Y.W. Chung, F. Ahmad, Y. Tang, S.C. Hockman, H.J. Kee, K. Berger, et al., White to beige conversion in PDE3B KO adipose tissue through activation of AMPK signaling and mitochondrial function, Sci. Rep. (2017) 740445.

[38] E.P. Mottillo, E.M. Desjardins, J.D. Crane, B.K. Smith, A.E. Green, S. Ducommun, et al., Lack of adipocyte AMPK exacerbates insulin resistance and hepatic steatosis through brown and beige adipose tissue function, Cell Metab. 24 (1) (2016) 118-129.

[39] K.M. Imran, D. Yoon, Y.S. Kim, A pivotal role of AMPK signaling in medicarpinmediated formation of brown and beige, BioFactors 44 (2) (2018) 168-179.

[40] E. Wahlberg, T. Karlberg, E. Kouznetsova, N. Markova, A. Macchiarulo, A.G. Thorsell, et al., Family-wide chemical profiling and structural analysis of PARP and tankyrase inhibitors, Nat. Biotechnol. 30 (3) (2012) 283-288.

[41] E. Kristof, Q.M. Doan-Xuan, A.K. Sarvari, A. Klusoczki, P. Fischer-Posovszky, M. Wabitsch, et al., Clozapine modifies the differentiation program of human adipocytes inducing browning, Transl. Psychiatry 6 (11) (2016) e963.

[42] P. Fischer-Posovszky, F.S. Newell, M. Wabitsch, H.E. Tornqvist, Human SGBS cells a unique tool for studies of human fat cell biology, Obes Facts 1 (4) (2008) 184-189.

[43] C. Elabd, C. Chiellini, M. Carmona, J. Galitzky, O. Cochet, R. Petersen, et al., Human multipotent adipose-derived stem cells differentiate into functional brown adipocytes, Stem Cells 27 (11) (2009) 2753-2760.

[44] M. Szántó, A. Brunyánszki, J. Márton, G. Vámosi, L. Nagy, T. Fodor, et al., Deletion of PARP-2 induces hepatic cholesterol accumulation and decrease in HDL levels, Biochem Biophys Acta - Mol. Basis Dis. 1842 (4) (2014) 594-602.

[45] L. Nagy, J. Marton, A. Vida, G. Kis, E. Bokor, S. Kun, et al., Glycogen phosphorylase inhibition improves beta cell function, Br. J. Pharmacol. 175 (2) (2018) 301-319.

[46] C.T. Rueden, J. Schindelin, M.C. Hiner, B.E. DeZonia, A.E. Walter, E.T. Arena, et al., Image J2: ImageJ for the next generation of scientific image data, BMC Bioinf. 18 (1) (2017) 529.

[47] E. Kristof, Q.M. Doan-Xuan, P. Bai, Z. Bacso, L. Fesus, Laser-scanning cytometry can quantify human adipocyte browning and proves effectiveness of irisin, Sci. Rep.
(2015) 512540

[48] O. Abdul-Rahman, E. Kristof, Q.M. Doan-Xuan, A. Vida, L. Nagy, A. Horvath, et al., AMP-activated kinase (AMPK) activation by AICAR in human white adipocytes derived from pericardial white adipose tissue stem cells induces a partial beige-like phenotype, PLoS One 11 (6) (2016) e0157644.

[49] Q.M. Doan-Xuan, A.K. Sarvari, P. Fischer-Posovszky, M. Wabitsch, Z. Balajthy, L. Fesus, et al., High content analysis of differentiation and cell death in human adipocytes, Cytometry A 83 (10) (2013) 933-943.

[50] E. Pirinen, E. Cantó, S.K. Jo, L. Morato, H. Zhang, K.J. Menzies, et al., Pharmacological inhibition of poly(ADP-Ribose) polymerases improves fitness and mitochondrial function in skeletal muscle, Cell Metab. 19 (6) (2014) 1034-1041.

[51] R. Cerutti, E. Pirinen, C. Lamperti, S. Marchet, A. Sauve, W. Li, et al., NAD + -dependent activation of sirt1 corrects the phenotype in a mouse model of mitochondrial disease, Cell Metab. 19 (6) (2014) 1042-1049.

[52] U. Kolthur-Seetharam, F. Dantzer, M.W. McBurney, G. de Murcia, P. Sassone-Corsi, Control of AIF-mediated cell death by the functional interplay of SIRT1 and PARP-1 in response to DNA damage, Cell Cycle 5 (8) (2006) 873-877.

[53] R. El Ramy, N. Magroun, N. Messadecq, L.R. Gauthier, F.D. Boussin, U. KolthurSeetharam, et al., Functional interplay between Parp-1 and SirT1 in genome integrity and chromatin-based processes, Cell. Mol. Life Sci. 66 (19) (2009) 3219-3234.

[54] A.S. Banks, N. Kon, C. Knight, M. Matsumoto, R. Gutierrez-Juarez, L. Rossetti, et al., SirT1 gain of function increases energy efficiency and prevents diabetes in mice, Cell Metab. 8 (4) (2008) 333-341.

[55] L. Qiang, H.V. Lin, J.Y. Kim-Muller, C.L. Welch, W. Gu, D. Accili, Proatherogenic abnormalities of lipid metabolism in SirT1 transgenic mice are mediated through Creb deacetylation, Cell Metab. 14 (6) (2011) 758-767.

[56] L. Qiang, L. Wang, N. Kon, W. Zhao, S. Lee, Y. Zhang, et al., Brown remodeling of white adipose tissue by SirT1-dependent deacetylation of ppargamma, Cell 150 (3) (2012) 620-632.

[57] J.R. Revollo, A.A. Grimm, S. Imai, The NAD biosynthesis pathway mediated by nicotinamide phosphoribosyltransferase regulates Sir2 activity in mammalian cells, J. Biol. Chem. 279 (49) (2004) 50754-50763.

[58] D.G. Hardie, B.E. Schaffer, A. Brunet, AMPK: an energy-sensing pathway with multiple inputs and outputs, Trends Cell Biol. 26 (3) (2016) 190-201.

[59] J.M. de Jong, O. Larsson, B. Cannon, J. Nedergaard, A stringent validation of mouse adipose tissue identity markers, Am. J. Physiol. Endocrinol. Metab. 308 (12) (2015) E1085-E1105.

[60] P. Seale, H.M. Conroe, J. Estall, S. Kajimura, A. Frontini, J. Ishibashi, et al., Prdm16 determines the thermogenic program of subcutaneous white adipose tissue in mice, J. Clin. Invest. 121 (1) (2011) 96-105.

[61] H. Wang, M. Shimoji, S.W. Yu, T.M. Dawson, V.L. Dawson, Apoptosis inducing factor and PARP-mediated injury in the MPTP mouse model of Parkinson's disease, Ann. N. Y. Acad. Sci. (2003) 991132-991139.

[62] S.A. Andrabi, H.C. Kang, J.F. Haince, Y.I. Lee, J. Zhang, Z. Chi, et al., Iduna protects the brain from glutamate excitotoxicity and stroke by interfering with poly(ADPribose) polymer-induced cell death, Nat. Med. 17 (6) (2011) 692-699.

[63] W. Ying, Y. Chen, C.C. Alano, R.A. Swanson, Tricarboxylic acid cycle substrates prevent PARP-mediated death of neurons and astrocytes, J. Cereb. Blood Flow Metab. 22 (7) (2002) 774-779.

[64] S. Diani-Moore, P. Ram, X. Li, P. Mondal, D.Y. Youn, A.A. Sauve, et al., Identification of the aryl hydrocarbon receptor target gene TiPARP as a mediator of suppression of hepatic gluconeogenesis by 2,3,7,8-tetrachlorodibenzo-p-dioxin and of nicotinamide as a corrective agent for this effect, J. Biol. Chem. 285 (50) (2010) 38801-38810.

[65] T.Y. Yeh, K.K. Beiswenger, P. Li, K.E. Bolin, R.M. Lee, T.S. Tsao, et al., Hypermetabolism, hyperphagia, and reduced adiposity in tankyrase-deficient mice, Diabetes (2009) 112476-112485.

[66] W.M. Shieh, J.C. Ame, M.V. Wilson, Z.Q. Wang, D.W. Koh, M.K. Jacobson, et al., Poly(ADP-ribose) polymerase null mouse cells synthesize ADP-ribose polymers, J. Biol. Chem. 273 (46) (1998) 30069-30072.

[67] V.C. Khanh, A.F. Zulkifli, C. Tokunaga, T. Yamashita, Y. Hiramatsu, O. Ohneda, Aging impairs beige adipocyte differentiation of mesenchymal stem cells via the reduced expression of Sirtuin 1, Biochem. Biophys. Res. Commun. 500 (3) (2018) 682-690.

[68] M. Garaulet, J.J. Hernandez-Morante, J. Lujan, F.J. Tebar, S. Zamora, Relationship between fat cell size and number and fatty acid composition in adipose tissue from different fat depots in overweight/obese humans, Int. J. Obes. (Lond). 30 (6) (2006) 899-905.

[69] E. Luche, C. Sengenes, E. Arnaud, P. Laharrague, L. Casteilla, B. Cousin, Differential hematopoietic activity in white adipose tissue depending on its localization, J. Cell. Physiol. 230 (12) (2015) 3076-3083.

[70] A. Roca-Rivada, J. Alonso, O. Al-Massadi, C. Castelao, J.R. Peinado, L.M. Seoane, et al., Secretome analysis of rat adipose tissues shows location-specific roles for each depot type, J. Proteom. 74 (7) (2011) 1068-1079.

[71] H.S. Sacks, J.N. Fain, S.W. Bahouth, S. Ojha, A. Frontini, H. Budge, et al., Adult epicardial fat exhibits beige features, J. Clin. Endocrinol. Metab. 98 (9) (2013) E1448-E1455.

[72] O. Zhu, S. Ghoshal, A. Rodrigues, S. Gao, A. Asterian, T.M. Kamenecka, et al., Adipocyte-specific deletion of Ip6k1 reduces diet-induced obesity by enhancing AMPK-mediated thermogenesis, J Clin Invest. 126 (11) (2016) 4273-4288.

[73] E.M. Desjardins, G.R. Steinberg, Emerging role of AMPK in brown and beige adipose tissue (BAT): implications for obesity, insulin resistance, and type 2 diabetes, Curr. Diab. Rep. 18 (10) (2018) 80

[74] L. Wu, L. Zhang, B. Li, H. Jiang, Y. Duan, Z. Xie, et al., AMP-activated protein kinase (AMPK) regulates energy metabolism through modulating thermogenesis in adipose 
tissue, Front. Physiol. 9 (2018) 122, https://doi.org/10.3389/fphys.2018.00122.

[75] C. Canto, Z. Gerhart-Hines, J.N. Feige, M. Lagouge, L. Noriega, J.C. Milne, et al, AMPK regulates energy expenditure by modulating NAD + metabolism and SIRT activity, Nature 458 (7241) (2009) 1056-1060.

[76] X. Luo, K.W. Ryu, D.S. Kim, T. Nandu, C.J. Medina, R. Gupte, et al., PARP-1 controls the adipogenic transcriptional program by PARylating C/EBPbeta and modulating its transcriptional activity, Mol. Cell 65 (2) (2017) 260-271, https://doi.org/10. 1016/j.molcel.2016.11.015.

[77] M.E. Smulson, V.H. Kang, J.M. Ntambi, D.S. Rosenthal, R. Ding C.M. Simbulan, Requirement for the expression of poly(ADP-ribose) polymerase during the early stages of differentiation of 3T3-L1 preadipocytes, as studied by antisense RNA in duction, J. Biol. Chem. 270 (1) (1995) 119-127.
[78] S. Erener, A. Mirsaidi, M. Hesse, A.N. Tiaden, H. Ellingsgaard, R. Kostadinova, et al., ARTD1 deletion causes increased hepatic lipid accumulation in mice fed a high-fat diet and impairs adipocyte function and differentiation, FASEB J. 26 (6) (2012) 2631-2638.

[79] P. Bai, S.M. Houten, A. Huber, V. Schreiber, M. Watanabe, B. Kiss, et al., Poly(ADP ribose) polymerase-2 controls adipocyte differentiation and adipose tissue function through the regulation of the activity of the retinoid $\mathrm{X}$ receptor/peroxisome proliferator-activated receptor-gamma heterodimer, J. Biol. Chem. 282 (52) (2007) 37738-37746.

[80] D. Huang, C. Yang, Y. Wang, Y. Liao, K. Huang, PARP-1 suppresses adiponectin expression through poly(ADP-ribosyl)ation of PPAR gamma in cardiac fibroblasts, Cardiovasc. Res. 81 (1) (2009) 98-107. 